Pacific Journal of Mathematics

THE CLASSIFICATION OF FLAT COMPACT COMPLETE 


\section{THE CLASSIFICATION OF \\ FLAT COMPACT COMPLETE SPACE-FORMS WITH METRIC OF SIGNATURE $(2,2)$}

\section{MING WANG}

Those flat compact complete space-forms with metric of signature $(2,2)$ are classified up to finite covers. The simply transitive subgroups of $R^{4} \rtimes \mathrm{SO}(2,2)$ are classified up to conjugation.

\section{Introduction.}

(1.1) If $\Gamma \subseteq R^{4} \rtimes \mathrm{SO}(2,2)$ and $\Gamma$ acts on $R^{p+q}$ freely and properly discontinuously with compact quotient, then $X=R^{p+q} / \Gamma$ is a flat compact complete space-form with metric of signature $(p, q)$. Recently D. Fried [3] has classified those flat compact complete spaceforms with metric of signature $(1,3)$ upto finite covers. Ravi S. Kulkarni pointed out that Fried's method can be applied to the case $(p, q)=(2,2)$. The basic idea of Fried's method is in the following theorem:

(1.2) THEOREM. Suppose $X$ is a flat compact complete space-form with fundamental group $\Gamma \subseteq R^{4} \rtimes \mathrm{SO}(2,2)$. Then there is a uniquely determined subgroup $H$ of $R^{4} \times \mathrm{SO}(2,2)$ that acts simply transitively on $R^{4}$ and $H \cap \Gamma=\pi$ has finite index in $\Gamma$.

(1.3) In $\S 2$ we classify those subgroups of $R^{4} \rtimes \mathrm{SO}(2,2)$ that act on $R^{4}$ simply transitively, up to the conjugacy of $R^{4} \rtimes \mathrm{O}(2,2)$. Every such subgroup, as a Lie group, is isomorphic to one of the following:

$$
\begin{gathered}
R^{4}, \quad R \times \mathrm{Nil}^{3}, \quad \mathrm{Nil}^{4}, \quad R \times\left\{R^{2} \rtimes\left(\begin{array}{cc}
e^{t} & 0 \\
0 & e^{-t}
\end{array}\right) ; t \in R\right\}, \\
R \times\left\{R^{2} \rtimes \mathrm{SO}(2)\right\} .
\end{gathered}
$$

All of them, except the last one, correspond to $\Gamma$ 's. Their uniform lattices are known, cf. [3] and [7].

(1.4) To prove Theorem (1.2), we first prove in $\S 3$ that $\Gamma$ is virtually solvable. This result confirms a conjecture by Milnor in a special case. In [6], it is conjectured that the fundamental group of a complete affinely flat manifold is virtually polycyclic. Our result, combined with 
Fried's result, shows that this conjecture is true for compact pseudoRiemannian 4-manifolds.

(1.5) In $\S 4$ we complete the proof of Theorem 1.2, using the theory of crystallographic hull developed by Fried and Goldman, cf. [4]. In $\S 5$, we give our classification. By comparing our list with Fried's, we obtain an interesting fact: as differential manifolds, they are the same coset spaces of the form $H / \Gamma$, where $H$ is a Lie group isomorphic to $R^{4}, R \times \mathrm{Nil}^{3}, \mathrm{Nil}^{4}$ or $R \times\left\{R^{2} \rtimes\left(\begin{array}{cc}e^{t} & 0 \\ 0 & e^{-t}\end{array}\right) ; t \in R\right\}$ and $\Gamma$ is a uniform lattice of $H$. These Lie groups have simply transitive representations as affine motions and when the signature is $(2,2)$ (resp. $(3,1)$ ), the images of the representations are $R^{4} \rtimes \mathrm{SO}(2,2)$ (resp. $\left.R^{4} \rtimes \mathrm{SO}(3,1)\right)$.

(1.6) Notations and some properties of $\mathrm{SO}(2,2)$ and $\mathrm{so}(2,2)$. Throughout this paper we will call $\left\{e_{i}\right\}, 1 \leq i \leq 4$, a standard basis s.t. the metric $Q$, w.r.t. this basis, has the form

$$
Q(v, v)=v_{1} v_{3}+v_{2} v_{4},
$$

where $v=\sum_{i=1}^{4} v_{i} e_{i}$. The full group of orientation-preserving isometries is $R^{4} \rtimes \mathrm{SO}(2,2)$ and

$$
\mathrm{SO}(2,2)=\left\{g \in \mathrm{SL}_{4}(R) ;{ }^{t} g\left(\begin{array}{cc}
0 & I \\
I & 0
\end{array}\right) g=\left(\begin{array}{cc}
0 & I \\
I & 0
\end{array}\right)\right\}
$$

where $I=\left(\begin{array}{ll}1 & 0 \\ 0 & 1\end{array}\right)$. The infinitesimal isometries are $R^{4} \rtimes \operatorname{so}(2,2)$ and

$$
\begin{aligned}
\operatorname{so}(2,2) & =\left\{X \in \mathrm{gl}_{4}(R) ;{ }^{t} X\left(\begin{array}{cc}
0 & I \\
I & 0
\end{array}\right)+\left(\begin{array}{ll}
0 & I \\
I & 0
\end{array}\right) X=0\right\} \\
& =\left\{\left(\begin{array}{cccc}
a_{11} & a_{12} & 0 & d \\
a_{21} & a_{22} & -d & 0 \\
0 & c & -a_{11} & -a_{21} \\
-c & 0 & -a_{12} & -a_{22}
\end{array}\right) ; a_{i j}, d, c \in R\right\} .
\end{aligned}
$$

(1.6.2) $\operatorname{so}(2,2)=L_{1} \oplus L_{2}$, where $L_{i} \simeq \operatorname{sl}_{2}(R), i=1,2$; and

$$
\begin{aligned}
& L_{1}=\left\{\left(\begin{array}{cccc}
a & b & \\
c & -a & & \\
& & -a & -c \\
& & -b & a
\end{array}\right) ; a, b, c \in R\right\}, \\
& L_{2}=\left\{\left(\begin{array}{cccc}
a^{\prime} & 0 & 0 & d^{\prime} \\
0 & a^{\prime} & -d^{\prime} & 0 \\
0 & c^{\prime} & -a^{\prime} & 0 \\
-c^{\prime} & 0 & 0 & -a^{\prime}
\end{array}\right) ; a^{\prime}, d^{\prime}, c^{\prime} \in R\right\} .
\end{aligned}
$$

$L_{1}, L_{2}$ are permuted by an element of $\mathrm{O}(2,2)$. 
(1.6.3) It is easy to show that any Cartan subalgebra of so $(2,2)$ is conjugate under $\mathrm{O}(2,2)$ to one of the following:

$$
\begin{aligned}
& \left\{\left(\begin{array}{cccc}
a & & & \\
& b & & \\
& & -a & \\
& & & -b
\end{array}\right) ; a, b, \in R\right\}, \\
& \left\{\left(\begin{array}{cccc}
0 & a & 0 & b \\
-a & 0 & -b & 0 \\
0 & b & 0 & a \\
-b & 0 & -a & 0
\end{array}\right) ; a, b, \in R\right\}, \\
& \left\{\left(\begin{array}{cccc}
a & b & & \\
-b & a & & \\
& & -a & b \\
& & -b & -a
\end{array}\right) ; a, b, \in R\right\} .
\end{aligned}
$$

An immediate corollary is

(1.6.4) If $X$ is in a Cartan subalgebra of $\operatorname{so}(2,2)$ and $\operatorname{det} X=0$, then $X$ must conjugate under $\mathrm{O}(2,2)$ to

$$
\left\{\left(\begin{array}{cccc}
a & & & \\
& 0 & & \\
& & -a & \\
& & & 0
\end{array}\right)\right\},
$$

or

$$
\left\{\left(\begin{array}{cccc}
0 & a & 0 & a \\
-a & 0 & -a & 0 \\
0 & a & 0 & a \\
-a & 0 & -a & 0
\end{array}\right)\right\}
$$

(1.7) We identify $\operatorname{Aff}(n)$, resp. aff $(n)$, with

$$
\left\{\left(\begin{array}{cc}
A & v \\
0 & a
\end{array}\right) ; A \in \mathrm{GL}_{4}(R), v \in R^{4}\right\},
$$

resp.

$$
\left\{\left(\begin{array}{cc}
X & v \\
0 & 0
\end{array}\right) ; X \in \mathrm{gl}_{4}(R), v \in R^{4}\right\},
$$

w.r.t. a given basis. Let $P_{l}$ be the natural homomorphism taking an affine transformation (or an infinitesimal affine transformation) to its linear part. Let $L(G)$ be the Lie algebra of a Lie group $G$ and $A(G)$ be the algebraic hull of $G$. We will need the following well-known lemma. 
(1.7.1) LemmA. If $G \subseteq \operatorname{Aff}(n)$ s.t. $G$ acts freely on $R^{n}$, then every $A \in P_{l}(G)$ has 1 as an eigenvalue.

(1.7.2) LemMA (Kostant and Sullivan, cf. [5]). If $G$ is as in (1.7.1), then every $A \in P_{l}(A(G))$ has 1 as an eigenvalue.

(1.7.3) Corollary. If $G$ is an in (1.7.1), then every $X \in$ $P_{l}\left(L(A(G))\right.$ or $X \in L\left(A\left(P_{l}(G)\right)\right.$ has 0 as an eigenvalue.

2. Simply transitive subgroups. We will classify subgroups of $R^{4} \rtimes$ $\mathrm{SO}(2,2)$ that act simply transitively on $R^{4}$. Our classification is up to the conjugation under $R^{4} \rtimes \mathrm{O}(2,2)$. It is well known that a simply transitive group of affine motions must be solvable, connected, simply connected and of dimension 4, cf. [1]. We will start from a special case when the groups are unipotent. The following lemma from Auslander and Scheuneman plays the key role in this section.

(2.1) LEMMA. Let $U$ be a nilpotent Lie group which has a faithful representation $\rho: U \rightarrow \operatorname{Aff}(n)$, let $\rho_{*}$ be the induced monomorphism of Lie algebras

$$
\rho_{*} L(U) \rightarrow\left\{\left(\begin{array}{cc}
X & v \\
0 & 0
\end{array}\right) ; X \in \mathrm{gl}_{n}(R), v \in R^{n}\right\}=\operatorname{aff}(n),
$$

and let $P_{l}$ be as in (1.7), let $P_{t}$ be the projection from an element in aff $(n)$ to its translation part. Then $\rho(U)$ acts on $R^{n}$ simply transitively if and only if

(1) $P_{l} \circ \rho_{*}(L(U))$ is nilpotent, and

(2) $P_{t} \circ \rho_{*}(L(U))$ is a linear isomorphism of $L(U)$ onto $R^{n}$.

For a proof, cf. [1]. So unipotent simply transitive subgroups are exactly the following $U$ 's s.t.

$$
L(U)=\left\{\left(\begin{array}{cc}
X(v) & v \\
0 & 0
\end{array}\right) ; v \in R^{n}\right\},
$$

where $X(v)$ is a linear function of $v$ and $P_{l}(L(U))=\left\{X(v) ; v \in R^{n}\right\}$ is nilpotent.

(2.3) LEMMA. There is a vector $v_{0} \in R^{4}$ such that

(i) $P_{l}(L(U))\left(v_{0}\right)=0$,

(ii) $Q\left(v_{0}, v_{0}\right)=0$. 
Proof. If $V=\left\{v \in R^{4} ; P_{l}(L(U)) v=0\right\}$, then $V^{\perp}$ is invariant. By Engel's Theorem on $V^{\perp}, V^{\perp}$ meets $V$.

Let $\left\{e_{i}\right\}$ be our standard basis. Then we choose $v_{0}=e_{1}$ since $\mathrm{O}(2,2)$ is transitive on $\{v ; Q(v, v)=0\} / v \sim t v$, where $t \in R-\{0\}$.

(2.4) CoROllaRY. W.r.t. the above standard basis, $X \in P_{l}(L(U))$ has the form

$$
X(v)=\left(\begin{array}{cccc}
0 & a & 0 & b \\
0 & 0 & -b & 0 \\
0 & 0 & 0 & 0 \\
0 & 0 & -a & 0
\end{array}\right),
$$

where $a=a(v)$ and $b=b(v)$ are linear functions of $v$.

To find $a(v)$ and $b(v)$, we compute the commutator of $L(U)$.

$$
\left[\left(\begin{array}{cc}
X(v) & v \\
0 & 0
\end{array}\right),\left(\begin{array}{cc}
X\left(v^{\prime}\right) & v^{\prime} \\
0 & 0
\end{array}\right)\right]=\left(\begin{array}{cc}
X\left(v^{\prime \prime}\right) & v^{\prime \prime} \\
0 & 0
\end{array}\right)
$$

where $v^{\prime \prime}=X(v) v^{\prime}-X\left(v^{\prime}\right) v, X\left(v^{\prime \prime}\right)=X(v) X\left(v^{\prime}\right)-X\left(v^{\prime}\right) X(v)=0$. So

$$
a\left(v^{\prime \prime}\right)=b\left(v^{\prime \prime}\right) \equiv 0
$$

Write

$$
a(v)=\sum_{i=1}^{4} a_{i} v_{i}, \quad b(v)=\sum_{i=1}^{4} b_{i} v_{i}
$$

Then we have

$$
0=\sum_{i=1}^{4} a_{i} v_{i}^{\prime \prime}, \quad 0=\sum_{i=1}^{4} b_{i} v_{i}^{\prime \prime},
$$

where $v_{i}^{\prime \prime}$ 's are linear functions of $a_{i}, b_{i}$ and $v_{i} v_{j}^{\prime}, 1 \leq i, j \leq 4$, and all coefficients of $v_{i} v_{j}^{\prime}$ must be zero. We obtain

(2.8) LEMMA.

(i) $a_{1}=b_{1}=0$,

(ii) $a_{2} b_{4}+a_{4}^{2}=0$,

(iii) $a_{2} b_{2}+a_{4} a_{2}=0$,

(iv) $b_{2} b_{4}+b_{4} a_{4}=0$,

(v) $b_{2}^{2}+b_{4} a_{2}=0$. 
(2.9) Corollary.

(i) $b_{4}\left(b_{2}+a_{4}\right)=0$,

(ii) $a_{2}\left(b_{2}+a_{4}\right)=0$,

(iii) $\left(b_{2}-a_{4}\right)\left(b_{2}+a_{4}\right)=0$.

(2.10) Now we can get some necessary conditions for the nontranslation unipotent simply transitive subgroups. If $b_{2}+a_{4} \neq 0$, then $b_{4}=a_{2}=0$. By (2.8) (ii) and (v), $b_{2}^{2}=a_{4}^{2}=0$ and we get a contradiction. So $b_{2}+a_{4}=0$, and we have three subcases:

(2.10.1) $b_{2}=a_{4}=b_{4}=a_{2}=0$, but $\left(a_{3}, b_{3}\right) \neq(0,0)$, i.e.,

$$
\left\{\begin{array}{l}
a(v)=a_{3} v_{3} \\
b(v)=b_{3} v_{3} .
\end{array}\right.
$$

(2.10.2) $b_{2}+a_{4}=0$ but $b_{2} \neq 0, a_{4} \neq 0$. Then by $(2.8) b_{4} \neq 0$, $a_{2} \neq 0$, i.e.

$$
\left\{\begin{array}{l}
a(v)=a_{2} v_{2}+a_{3} v_{3}+a_{4} v_{4} \\
b(v)=b_{2} v_{2}+b_{3} v_{3}+b_{4} v_{4}
\end{array}\right.
$$

(2.10.3) $b_{2}=0, a_{4}=0,\left(a_{2}, b_{4}\right) \neq(0,0)$. By $(2.8), b_{4} a_{2}=0$, so

$$
\left\{\begin{array}{l}
a(v)=a_{2} v_{2}+a_{3} v_{3} \\
b(v)=b_{3} v_{3}
\end{array}\right.
$$

or

$$
\left\{\begin{array}{l}
a(v)=a_{3} v_{3} \\
b(v)=b_{3} v_{3}+b_{4} v_{4}
\end{array}\right.
$$

(2.11) Theorem. Up to conjugacy under $R^{4} \rtimes \mathrm{O}(2,2)$, the nontranslation unipotent simply transitive groups $U$ of $R^{4} \rtimes \mathrm{SO}(2,2)$, have the following Lie algebras:

$$
L(U)=\left\{\left(\begin{array}{cc}
X(v) & v \\
0 & 0
\end{array}\right) ; v \in R^{4}\right\}
$$

where

$$
X(v)=\left(\begin{array}{cccc}
0 & a(v) & 0 & b(v) \\
0 & 0 & -b(v) & 0 \\
0 & 0 & 0 & 0 \\
0 & 0 & -a(v) & 0
\end{array}\right)
$$


$a(v)$ and $b(v)$ are listed in the following table:

\begin{tabular}{|c|c|c|c|}
\hline Type of $L(U)$ & $a(v)$ & $b(v)$ & $\begin{array}{c}\text { isomorphism type as an } \\
\text { abstract Lie algebra }\end{array}$ \\
\hline I-1 & $v_{3}$ & $v_{3}$ & $N_{3} \oplus R$ \\
\hline I-2 & $v_{3}$ & $-v_{3}$ & $N_{3} \oplus R$ \\
\hline I-3 & $v_{3}$ & 0 & $N_{3} \oplus R$ \\
\hline II-1 & $v_{2}+v_{4}+t v_{3},(t \geq 0)$ & $-v_{2}-v_{4}$ & $N_{4}$ \\
\hline II-2 & $-v_{2}+v_{4}+t v_{3},(t \geq 0)$ & $-v_{2}+v_{4}$ & $N_{4}$ \\
\hline II-3 & $v_{2}$ & $v_{3}$ & $N_{4}$ \\
\hline
\end{tabular}

The equivalence classes are uniquely determined by the type of $L(U)$ and the parameter $t$ (in Type II).

Proof. The discussion of the conjugacy under $R^{4} \rtimes \mathrm{O}(2,2)$ is long and tedious. We will only write down a brief one for subcase (2.10.2). We give the following lemma without proof.

(2.11.1) LEMMA. If $a(v) \not \equiv 0, b(v) \not \equiv 0, a^{\prime}\left(v^{\prime}\right) \not \equiv 0, b^{\prime}\left(v^{\prime}\right) \not \equiv 0$, and if there is a matrix $A=\left(a_{i j}\right) \in \mathrm{O}(2,2)$ such that

$$
A^{-1}\left(\begin{array}{cccc}
0 & a(v) & 0 & b(v) \\
0 & 0 & -b(v) & 0 \\
0 & 0 & 0 & 0 \\
0 & 0 & -a(v) & 0
\end{array}\right) A=\left(\begin{array}{cccc}
0 & a^{\prime}\left(v^{\prime}\right) & 0 & b^{\prime}\left(v^{\prime}\right) \\
0 & 0 & -b^{\prime}\left(v^{\prime}\right) & 0 \\
0 & 0 & 0 & 0 \\
0 & 0 & -a^{\prime}\left(v^{\prime}\right) & 0
\end{array}\right)
$$

then either

(1)

$$
\left\{\begin{aligned}
a^{\prime}\left(v^{\prime}\right)= & \frac{a_{2} a_{22}^{2}}{a_{11}} v_{2}^{\prime}+\left\{\frac{a_{22} a_{23}}{a_{11}} a_{2}+\frac{a_{22}}{a_{11}^{2}} a_{3}+\frac{a_{22} a_{43}}{a_{11}} a_{4}\right\} v_{3}^{\prime} \\
& +\frac{a_{4}}{a_{11}} v_{4}^{\prime} \\
b^{\prime}\left(v^{\prime}\right)= & \frac{b_{2}}{a_{11}} v_{2}^{\prime}+\left\{\frac{a_{23}}{a_{11} a_{22}} b_{2}+\frac{1}{a_{11}^{2} a_{22}} b_{3}+\frac{a_{43}}{a_{11} a_{22}} b_{4}\right\} v_{3}^{\prime} \\
& +\frac{b_{4}}{a_{11} a_{22}^{2}} v_{4}^{\prime}
\end{aligned}\right.
$$


where $a_{11} a_{22} \neq 0$; or

$$
\left\{\begin{aligned}
a^{\prime}\left(v^{\prime}\right)= & \frac{b_{4} a_{42}^{2}}{a_{11}} v_{2}^{\prime}+\left\{\frac{a_{42} a_{23}}{a_{11}} b_{2}+\frac{a_{42}}{a_{11}^{2}} b_{3}+\frac{a_{42} a_{43}}{a_{11}} b_{4}\right\} v_{3}^{\prime} \\
& +\frac{b_{2}}{a_{11}} v_{4}^{\prime} \\
b^{\prime}\left(v^{\prime}\right)= & \frac{a_{4}}{a_{11}} v_{2}^{\prime}+\left\{\frac{a_{23}}{a_{11} a_{42}} a_{2}+\frac{1}{a_{11}^{2} a_{42}} a_{3}+\frac{a_{43}}{a_{11} a_{42}} a_{4}\right\} v_{3}^{\prime} \\
& +\frac{a_{4}}{a_{11} a_{41}^{2}} v_{4}^{\prime}
\end{aligned}\right.
$$

where $a_{11} a_{42} \neq 0$.

Write $a^{\prime}\left(v^{\prime}\right)=\sum_{i=2}^{4} a_{i}^{\prime} v_{i}^{\prime}$ and $b^{\prime}\left(v^{\prime}\right)=\sum_{i=2}^{4} b_{i}^{\prime} v_{i}^{\prime}$, then from $(2.11 .1)$

$$
a_{2}^{\prime} b_{4}^{\prime}=a_{4}^{\prime} b_{2}^{\prime}=\frac{a_{4} b_{2}}{a_{11}^{2}}=-\frac{a_{4}^{2}}{a_{11}^{2}}<0,
$$

since $a_{4}=-b_{2} \neq 0$. So we can choose $a_{11}$ such that $a_{2}^{\prime} b_{4}^{\prime}=a_{4}^{\prime} b_{2}^{\prime}=$ -1 , i.e. $a_{4} / a_{11}= \pm 1$. Next we use (1) (resp. (2)) if $a_{4} / a_{11}=1$ (resp. -1 ), and choose $a_{22}$ (resp. $a_{42}$ ) to reduce

$$
\left(\begin{array}{ll}
a_{2}^{\prime} & a_{4}^{\prime} \\
b_{2}^{\prime} & b_{4}^{\prime}
\end{array}\right)
$$

to

$$
\left(\begin{array}{cc}
1 & 1 \\
-1 & -1
\end{array}\right) \text { if } a_{2} a_{4}>0
$$

or

$$
\left(\begin{array}{ll}
-1 & 1 \\
-1 & 1
\end{array}\right) \text { if } a_{2} a_{4}<0
$$

Now $a_{3}^{\prime}, b_{3}^{\prime}$ have the form

$$
\left\{\begin{array} { l } 
{ a _ { 3 } ^ { \prime } = z _ { 1 } + \frac { a _ { 2 2 } } { a _ { 1 1 } ^ { 2 } } a _ { 3 } } \\
{ b _ { 3 } ^ { \prime } = \pm z _ { 1 } + \frac { 1 } { a _ { 1 1 } ^ { 2 } a _ { 2 2 } } b _ { 3 } , }
\end{array} , \text { or } \left\{\begin{array}{l}
a_{3}^{\prime}=z_{2}+\frac{a_{42}}{a_{11}^{2} b_{3}} \\
b_{3}^{\prime}= \pm z_{2}+\frac{1}{a_{11}^{2} a_{42}} a_{3},
\end{array}\right.\right.
$$

where $z_{1}$ (resp. $z_{2}$ ) depends on $a_{23}, a_{43}$ (resp. $a_{23}, a_{43}$ ) and $z_{i}$, $i=1,2$ can assume any real number. We can choose $z_{i}$ so that $b_{3}^{\prime}=0$ and we can choose the sign of $a_{22}$ (resp. $a_{42}$ ) so that $a_{3}^{\prime} \geq 0$. So we can find an $A \in \mathrm{O}(2,2)$ such that

$$
\left(\begin{array}{cc}
A^{-1} & 0 \\
0 & 1
\end{array}\right)\left(\begin{array}{cc}
X & v \\
0 & 0
\end{array}\right)\left(\begin{array}{cc}
A & 0 \\
0 & 1
\end{array}\right)
$$


is of Type II-1 or Type II-2. We can replace $\left(\begin{array}{cc}A & 0 \\ 0 & 1\end{array}\right)$ by $\left(\begin{array}{cc}A & w \\ 0 & 1\end{array}\right)$ and show that the translation part doesn't contribute to the classification.

We omit the rest of the proof.

(2.12) To handle the general case, namely when the simply transitive group of affine motion is non-unipotent solvable, we need the following lemma from Auslander, cf. [1].

(2.12.1) LemMA. Let $H$ be an $n$-dimensional, connected, simply connected, solvable Lie group acting simply transitively as affine motions on $R^{n}$. Let $A(H)$ be the algebraic hull of $H$ and let $U$ be the unipotent radical of $A(H)$. Then $U$ operates simply transitively as affine motions on $R^{n}$.

Now all such nontranslation $U$ 's are known from (2.11), and we'll study them first.

(2.12.2) Lemma. Let $H, U$ be as in (2.12.1) and assume that $U$ is not the translation group $T$. Then $H=U$.

Proof. W.r.t. the standard basis $\left\{e_{i}\right\}, 1 \leq i \leq 4$, we know

$$
L\left(P_{l}(U)\right)=\left\{\left(\begin{array}{cccc}
0 & a(v) & 0 & b(v) \\
0 & 0 & -b(v) & 0 \\
0 & 0 & 0 & 0 \\
0 & 0 & -a(v) & 0
\end{array}\right) ; v \in R^{4}\right\}
$$

Notice that $A(H)$ is contained in the normalizer of $U$, we have

$$
[L(A(H)), L(U)] \subseteq L(U), \quad\left[L\left(P_{l}(A(H))\right), L\left(P_{l}(U)\right)\right] \subseteq L\left(P_{l}(U)\right)
$$

Since for

$$
Y=\left(\begin{array}{cccc}
a_{11} & a_{12} & 0 & d \\
a_{21} & a_{22} & -d & 0 \\
0 & c & -a_{11} & -a_{21} \\
-c & 0 & -a_{12} & -a_{22}
\end{array}\right), \quad X=\left(\begin{array}{cccc}
0 & a(v) & 0 & b(v) \\
0 & 0 & -b(v) & 0 \\
0 & 0 & 0 & 0 \\
0 & 0 & -a(v) & 0
\end{array}\right),
$$

we have

$[Y, X]$

$$
=\left(\begin{array}{cccc}
-a(v) a_{21}+b(v) c & a(v)\left(a_{11}-a_{22}\right) & 0 & b(v)\left(a_{11}+a_{22}\right) \\
0 & a(v) a_{21}+b(v) c & -b(v)\left(a_{11}+a_{22}\right) & 0 \\
0 & 0 & a(v) a_{21}-b(v) c & 0 \\
0 & 0 & -a(v)\left(a_{11}-a_{22}\right) & -a(v) a_{21}-b(v) c
\end{array}\right) .
$$


So

$$
\left\{\begin{array}{c}
-a(v) a_{21}+b(v) c=0 \\
a(v) a_{21}+b(v) c=0
\end{array}\right.
$$

i.e.

$$
\left\{\begin{array}{l}
a(v) a_{21}=0 \\
b(v) c=0
\end{array}\right.
$$

for any $a_{21}, c, a(v)$ and $b(v), v \in R^{4}$.

By (2.11), we can always find a $v$ so that $a(v) \neq 0$, so we must have $a_{21}=0$. Similarly $c=0$, unless $b(v) \equiv 0$. So we have two cases.

Case 1. Type of $L(U)$ is I-1, I-2 or II.

$L\left(P_{l}(A(H))\right)$ is contained in

$$
\left\{\left(\begin{array}{cccc}
a_{11} & a_{12} & 0 & d \\
0 & a_{22} & -d & 0 \\
0 & 0 & -a_{11} & 0 \\
0 & 0 & -a_{12} & -a_{22}
\end{array}\right) ; a_{11}, a_{12}, a_{22}, d \in R\right\}
$$

Case 2. Type of $L(U)$ is I-3. $L\left(P_{l}(A(H))\right)$ is contained in

$$
\left\{\left(\begin{array}{cccc}
a_{11} & a_{12} & 0 \\
0 & a_{22} & 0 & 0 \\
0 & c & -a_{11} & 0 \\
-c & 0 & -a_{12} & -a_{22}
\end{array}\right) ; a_{11}, a_{12}, a_{22}, c \in R\right\}
$$

It's easy to show that matrices in Case 1 and Case 2 are conjugate under $\mathrm{O}(2,2)$. We will only write down a proof for Case 1; a proof for Case 2 can be obtained similarly.

Again let $Y \in L\left(P_{l}(A(H))\right)$. Then by (1.7.3) det $Y=0$, so $a_{11} a_{22}=$ 0 , i.e. $a_{11}=0$ or $a_{22}=0$.

If $a_{22}=0$, then an element in $L(A(H))$ has the form

$$
\left(\begin{array}{ll}
Y & v \\
0 & 0
\end{array}\right)=\left(\begin{array}{ccccc}
a_{11} & a_{12} & 0 & d(v) & v_{1} \\
0 & 0 & -d & 0 & v_{2} \\
0 & 0 & -a_{11} & 0 & v_{3} \\
0 & 0 & -a_{12} & 0 & v_{4} \\
0 & 0 & 0 & 0 & 0
\end{array}\right), \quad \text { for some } v=\left(\begin{array}{l}
v_{1} \\
v_{2} \\
v_{3} \\
v_{4}
\end{array}\right)
$$


By subtracting an element $\left(\begin{array}{cc}X(v) & v \\ 0 & 0\end{array}\right) \in L(U)$, we have

$$
\begin{aligned}
& \left(\begin{array}{cc}
Y-X(v) & 0 \\
0 & 0
\end{array}\right) \\
& \quad\left(\begin{array}{ccccc}
a_{11} & a_{12}-a(v) & 0 & d-b(v) & 0 \\
0 & 0 & -d+b(v) & 0 & 0 \\
0 & 0 & -a_{11} & 0 & 0 \\
0 & 0 & -a_{12}+a(v) & 0 & 0 \\
0 & 0 & 0 & 0 & 0
\end{array}\right) \in L(A(H)) .
\end{aligned}
$$

For any $\left(\begin{array}{cc}X\left(v^{\prime}\right) & v^{\prime} \\ 0 & 0\end{array}\right) \in L(U)$, we have

$$
\begin{aligned}
& {\left[\left(\begin{array}{cc}
Y-X(v) & 0 \\
0 & 0
\end{array}\right),\left(\begin{array}{cc}
X\left(v^{\prime}\right) & v^{\prime} \\
070 &
\end{array}\right)\right]} \\
& =\left(\begin{array}{ccccc}
0 & a_{11} a\left(v^{\prime}\right) & 0 & a_{11} b\left(v^{\prime}\right) & a_{11} v_{1}^{\prime}+\left(a_{12}-a(v)\right) v_{2}^{\prime}+(d-b(v)) v_{4}^{\prime} \\
0 & 0 & -a_{11} b\left(v^{\prime}\right) & 0 & -(d-b(v)) v_{3}^{\prime} \\
0 & 0 & 0 & 0 & -a_{11} v_{3}^{\prime} \\
0 & 0 & -a_{11} a\left(v^{\prime}\right) & 0 & -\left(a_{12}-a(v)\right) v_{3}^{\prime} \\
0 & 0 & 0 & 0 & 0
\end{array}\right) \in L(U) .
\end{aligned}
$$

But we know that

$$
\left(\begin{array}{ccccc}
0 & a_{11} a\left(v^{\prime}\right) & 0 & a_{11} b\left(v^{\prime}\right) & a_{11} v_{1}^{\prime} \\
0 & 0 & -a_{11} b\left(v^{\prime}\right) & 0 & a_{11} v_{2}^{\prime} \\
0 & 0 & 0 & 0 & a_{11} v_{3}^{\prime} \\
0 & 0 & -a_{11} a\left(v^{\prime}\right) & 0 & a_{11} v_{4}^{\prime} \\
0 & 0 & 0 & 0 & 0
\end{array}\right) \in L(U) .
$$

So we have

(1) $a_{11} v_{1}^{\prime}=a_{11} v_{1}^{\prime}+\left(a_{12}-a(v)\right) v_{2}^{\prime}+(d-b(v)) v_{4}^{\prime}$;

(2) $a_{11} v_{2}^{\prime}=-(d-b(v)) v_{3}^{\prime}$;

(3) $a_{11} v_{3}^{\prime}=-a_{11} v_{3}^{\prime}$;

(4) $a_{11} v_{4}^{\prime}=-\left(a_{12}-a(v)\right) v_{3}^{\prime}$.

From (3) we get $a_{11}=0$. Then (2), resp. (4), implies $d=b(v)$, resp. $a_{12}=a(v)$, i.e. $Y=X(v)$. So $\left(\begin{array}{ll}Y & v \\ 0 & 0\end{array}\right) \in L(U)$.

If $a_{11}=0$, let $\left(\begin{array}{ll}Y & v \\ 0 & 0\end{array}\right) \in L(A(H))$. By subtracting an element $\left(\begin{array}{cc}X(v) & v \\ 0 & 0\end{array}\right) \in L(U)$, we have

$$
\begin{aligned}
& \left(\begin{array}{cc}
Y-X(v) & 0 \\
0 & 0
\end{array}\right) \\
& \quad\left(\begin{array}{ccccc}
0 & a-12-a(v) & 0 & d-b(v) & 0 \\
0 & a_{22} & -(d-b(v)) & 0 & 0 \\
0 & 0 & 0 & 0 & 0 \\
0 & 0 & -\left(a_{12}-a(v)\right) & -a_{22} & 0 \\
0 & 0 & 0 & 0 & 0
\end{array}\right) \in L(A(H)) .
\end{aligned}
$$


Then for any $\left(\begin{array}{cc}X\left(v^{\prime}\right) & v^{\prime} \\ 0 & 0\end{array}\right) \in L(U)$, we have

$$
\begin{aligned}
& {\left[\left(\begin{array}{cc}
Y-X(v) & 0 \\
0 & 0
\end{array}\right),\left(\begin{array}{cc}
X\left(v^{\prime}\right) & v^{\prime} \\
0 & 0
\end{array}\right)\right]} \\
& \quad=\left(\begin{array}{ccccc}
0 & -a_{22} a\left(v^{\prime}\right) & 0 & a_{22} b\left(v^{\prime}\right) & \left(a_{12}-a(v)\right) v_{2}^{\prime}+(d-b(v)) v_{4}^{\prime} \\
0 & 0 & -a_{22} b\left(v^{\prime}\right) & 0 & a_{22} v_{2}^{\prime}-(d-b(v)) v_{3}^{\prime} \\
0 & 0 & 0 & 0 & 0 \\
0 & 0 & a_{22} a\left(v^{\prime}\right) & 0 & -\left(a_{12}-a(v)\right) v_{3}^{\prime}-a_{22} v_{4}^{\prime} \\
0 & 0 & 0 & 0 & 0
\end{array}\right) \in L(U) .
\end{aligned}
$$

Let $a(v)=\sum a_{i} v_{i}, b(v)=\sum b_{i} v_{i}, 2 \leq i \leq 4$ as in (2.6) $\left(a_{1}=\right.$ $\left.b_{1}=0\right)$ and let

$$
v^{\prime \prime}=\left(\begin{array}{c}
\left(a_{12}-a(v)\right) v_{2}^{\prime}+(d-b(v)) v_{4}^{\prime} \\
a_{22} v_{2}^{\prime}-(d-b(v)) v_{3}^{\prime} \\
0 \\
-\left(a_{12}-a(v)\right) v_{3}^{\prime}-a_{22} v_{4}^{\prime}
\end{array}\right)
$$

Then

$$
\left\{\begin{array}{c}
-a_{22} a\left(v^{\prime}\right)=a\left(v^{\prime \prime}\right)=a_{2}\left(a_{22} v_{2}^{\prime}-(d-b(v)) v_{3}^{\prime}\right) \\
+a_{4}\left(-\left(a_{12}-a(v)\right) v_{3}^{\prime}-a_{22} v_{4}^{\prime}\right) \\
a_{22} b\left(v^{\prime}\right)=b\left(v^{\prime \prime}\right)=b_{2}\left(a_{22} v_{2}^{\prime}-(d-b(v)) v_{3}^{\prime}\right) \\
+b_{4}\left(-\left(a_{12}-a(v)\right) v_{3}^{\prime}-a_{22} v_{4}^{\prime}\right)
\end{array}\right.
$$

i.e.

$$
\left\{\begin{aligned}
-a_{22}\left(a_{2} v_{2}^{\prime}+a_{3} v_{3}^{\prime}+a_{4}+v_{4}^{\prime}\right)= & a_{2}\left(a_{22} v_{2}^{\prime}-(d-b(v)) v_{3}^{\prime}\right) \\
& +a_{4}\left(-\left(a_{12}-a(v)\right) v_{3}^{\prime}-a_{22} v_{4}^{\prime}\right), \\
a_{22}\left(b_{2} v_{2}^{\prime}+b_{3} v_{3}^{\prime}+b_{4} v_{4}^{\prime}\right)= & b_{2}\left(a_{22} v_{2}^{\prime}-(d-b(v)) v_{3}^{\prime}\right) \\
+ & b_{4}\left(-\left(a_{12}-a(v)\right) v_{3}^{\prime}-a_{22} v_{4}^{\prime}\right),
\end{aligned}\right.
$$

i.e.

$$
\left\{\begin{array}{l}
2 a_{22} a_{2} v_{2}^{\prime}+\left(a_{3} a_{22}-a_{4}\left(a_{12}-a(v)\right)-a_{2}(d-b(v))\right) v_{3}^{\prime}=0 \\
2 a_{22} b_{4} v_{4}^{\prime}+\left(b_{3} a_{22}+b_{4}\left(a_{12}-a(v)\right)+b_{2}(d-b(v))\right) v_{3}^{\prime}=0
\end{array}\right.
$$

Letting $v_{i}^{\prime}$ 's vary, we have
(1) $a_{22} a_{2}=0$;
(2) $a_{22} b_{4}=0$
(3) $a_{3} a_{22}-a_{4}\left(a_{12}-a(v)\right)-a_{2}(d-b(v))=0$;
(4) $b_{3} a_{22}+b_{4}\left(a_{12}-a(v)\right)+b_{2}(d-b(v))=0$. 
If $a_{22} \neq 0$, we must have $a_{2}=b_{4}=0$ by (1) and (2). According to (2.10), this implies $b_{2}=a_{4}=b_{4}=a_{2}=0$. Then (3) and (4) lead to

$$
\left\{\begin{array}{l}
a_{3} a_{22}=0, \\
b_{3} a_{22}=0,
\end{array}\right.
$$

i.e. $a_{3}=b_{3}=0$. So $U=T$, and we have a contradiction. So $a_{22}=0$. We always have $a_{11}=a_{22}=0$, i.e. $A(H)$ is unipotent; so $H$ is unipotent. But any unipotent connected Lie group is Zariski closed, so $H=A(H)$. $U$, as the unipotent radical of $H$ must be $H$ itself.

(2.12.3) Now consider the case when the unipotent radical $A(H)$ is precisely the group $T$ of translations of $R^{4}$. Suppose $H \neq T$, i.e. $H$ is not unipotent.

(2.12.3.1) Lemma. $P_{l}(H)$ is abelian.

Proof. $\left.P_{l}(H) \simeq H / \operatorname{Ker}\left(\left.P_{l}\right|_{H}\right)\right)=H /(H \cap T) \subseteq A(H) / T$, but $A(H) / T$ is abelian (cf. [2], $A(H) / U(H)$ is abelian, since $A(H)$ is solvable and algebraic).

(2.12.3.2) Lemma. $\operatorname{dim} P_{l}(H)=1 ; P_{l}(H)$ is diagnolizable in $\mathbf{C}$.

Proof. $P_{l}(H)$ is a connected abelian subgroup of $\mathrm{SO}_{0}(2,2)$, so $\operatorname{dim} P_{l}(H) \leq 2$. By (1.7.3) det $X=0$ for every $X \in L\left(P_{l}(H)\right)$, i.e. 0 is an eigenvalue of $X$. Since $X \in \operatorname{so}(2,2)$, so

$$
X=\left(\begin{array}{cccc}
a_{11} & a_{12} & 0 & d \\
a_{21} & a_{22} & -d & 0 \\
0 & c & -a_{11} & -a_{21} \\
-c & 0 & -a_{12} & -a_{22}
\end{array}\right),
$$

and

$$
\begin{aligned}
\operatorname{det}(X-\lambda I)= & \lambda^{4}+\left(2 d c-2 a_{12} a_{21}-a_{11}^{2}-a_{22}^{2}\right) \lambda^{2} \\
& +\left(-a_{11} a_{22}+a_{12} a_{21}+d c\right)^{2} \\
= & \lambda^{4}+\left\{-4 a_{12} a_{21}-\left(a_{11}-a_{12}\right)^{2}\right\} \lambda^{2},
\end{aligned}
$$

since 0 is an eigenvalue. So the eigenvalues of $X$ are $\{0,0,0,0\}$ or $\{0,0, \lambda,-\lambda\}, \lambda \neq 0, \lambda \in R$ or $\sqrt{-1} R$. If $\operatorname{dim} P_{l}(H)=2$, then by $(1.6 .2) \operatorname{so}(2,2)=L_{1} \oplus L_{2}, L_{i} \simeq \operatorname{sl}_{2}(R)$. So $L\left(P_{l}(H)\right)=$ $R X_{1}+R X_{2}$ where $X_{i} \in L_{i}, \quad i=1,2$. But by (1.6.2) 


$$
\begin{aligned}
& \operatorname{det}\left(X_{1}-\lambda I\right)=\lambda^{4}+2\left(a^{2}+b c\right) \lambda^{2}+\left(a^{2}+b c\right)^{2}, \text { and } \\
& \operatorname{det}\left(X_{2}-\lambda I\right)=\lambda^{4}+2\left(b^{\prime} c^{\prime}-a^{\prime 2}\right) \lambda^{2}+\left(b^{\prime} c^{\prime}-a^{\prime 2}\right)^{2} .
\end{aligned}
$$

So zero is an eigenvalue of $X_{i}, i=1,2$, if and only if all the eigenvalues of $X_{i}$ are zero. This means $P_{l}(H)$ is unipotent and leads to a contradiction. So $\operatorname{dim} P_{l}(H)=1, L\left(P_{l}(H)\right)=R X$ and $X$ has eigenvalues $\{0,0, \lambda,-\lambda\}, \lambda \neq 0, \lambda \in R$ or $\sqrt{-1} R$. Since $X$ is an infinitesimal isometry, it is diagnolizable.

(2.12.3.3) Corollary. $L\left(P_{l}(H)\right)$ is contained in a Cartan subalgebra of $\operatorname{so}(2,2)$ and is conjugate under $\mathrm{O}(2,2)$ to

$$
\begin{aligned}
& \left(\begin{array}{cccc}
a & & & 0 \\
& 0 & & \\
0 & & -a & \\
0 & & & 0
\end{array}\right) \\
& \left(\begin{array}{cccc}
0 & a & 0 & a \\
-a & 0 & -a & 0 \\
0 & a & 0 & a \\
-a & 0 & -a & 0
\end{array}\right) .
\end{aligned}
$$

Proof. By (1.6.4).

Since $H$ is simply transitive, the map $P_{t}: L(H) \rightarrow R^{4}$ is a linear isomorphism, so in (2.12.3.3) we have $a=\sum_{i=1}^{4} a_{i} v_{i}$, where

$$
v=\left(\begin{array}{l}
v_{1} \\
v_{2} \\
v_{3} \\
v_{4}
\end{array}\right)
$$

is the corresponding translation part. Since $T$ is the unipotent radical of $A(H)$, we have $[L(H), L(H)] \subseteq L(T)=R^{4}$. By computing the commutator and using the fact that $H$ is simply transitive, we must have $a(v)=a_{2} v_{2}+a_{4} v_{4},\left(a_{2}, a_{4}\right) \neq(0,0)$ in Case $(1)$ and $a(v)=$ $a_{1}\left(v_{1}-v_{3}\right)+a_{2}\left(v_{2}-v_{4}\right),\left(a_{1}, a_{2}\right) \neq(0,0)$ in Case (2). Finally, by considering the conjugation under $R^{4} \rtimes \mathrm{O}(2,2)$, we get

(2.12.4) THEOREM. If $H \subseteq R^{4} \rtimes \mathrm{SO}(2,2)$ acts simply transitively on $R^{4}$ and $H$ is not unipotent, then $H$ is conjugate under $R^{4} \rtimes \mathrm{O}(2,2)$ 
to one of the following:

(i) Type III-1:

$$
\left(\begin{array}{cccc}
a(v) & & & 0 \\
& 0 & & \\
& & -a(v) & \\
0 & & & 0
\end{array}\right)\left(\begin{array}{l}
v \\
\end{array}\right)
$$

where $a(v)=t v_{2}+v_{4}, t>0$ and $L(H) \simeq R \oplus\left\{R^{2} \rtimes R\left(\begin{array}{cc}1 & 0 \\ 0 & -1\end{array}\right)\right\}$.

(ii) Type III-2:

$$
\left(\begin{array}{cccc}
0 & a(v) & 0 & a(v) \\
-a(v) & 0 & -a(v) & 0 \\
0 & a(v) & 0 & a(v) \\
-a(v) & 0 & -a(v) & 0
\end{array}\right)\left(\begin{array}{l}
v \\
\end{array}\right)
$$

where $a(v)=t\left(v_{1}-v_{3}\right), t>0$ and $L(H)=R \oplus\left\{R^{2} \rtimes R\left(\begin{array}{cc}0 & 1 \\ -1 & 0\end{array}\right)\right\}$. The type and the parameter $t$ determine the equivalence classes uniquely.

(2.13) Combining (2.11) with (2.12.4) and denoting $H=T_{4}$ as Type 0 , we complete the classification of simply transitive subgroups of $R^{4} \rtimes \mathrm{SO}(2,2)$. We summarize our result in the following table. We denote

$$
\begin{aligned}
& A(a, b, v)=\left\{\left(\left(\begin{array}{cccc}
0 & a(v) & 0 & b(v) \\
0 & 0 & -b(v) & 0 \\
0 & 0 & 0 & 0 \\
0 & 0 & -a(v) & 0
\end{array}\right) v\left(v \in R^{4}\right\}\right.\right. \\
& B(a, v)=\left\{\left(\left(\begin{array}{llll}
a(v) & & & 0 \\
& 0 & & \\
& & -a(v) & \\
0 & & 0 & 0
\end{array}\right), v \in R^{4}\right\}\right. \text {, } \\
& C(a, v)=\left\{\left(\left(\begin{array}{cccc}
0 & a(v) & 0 & a(v) \\
-a(v) & 0 & -a(v) & 0 \\
0 & a(v) & 0 & a(v) \\
-a(v) & 0 & -a(v) & 0
\end{array}\right) ; v \in R^{4}\right\}\right.
\end{aligned}
$$


Table of equivalence classes of simply transitive subgroups of $R^{4} \rtimes$ $\mathrm{SO}(2,2)$ (given in the form of subalgebras of aff $(n)$ w.r.t. a standard basis).

\begin{tabular}{|c|c|c|}
\hline $\begin{array}{l}\text { type of } \\
L(H)\end{array}$ & affine form of $L(H)$ & $\begin{array}{c}\text { isomorphism type } \\
\text { as abstract } \\
\text { Lie algebra }\end{array}$ \\
\hline 0 & $\left\{\left(\begin{array}{ll}0 & v \\
0 & 0\end{array}\right) ; v \in R^{4}\right\}$ & $R^{4}$ \\
\hline I-1 & $A(a, b, v),\left\{\begin{array}{l}a(v)=v_{3} \\
b(v)=v_{3}\end{array}\right.$ & $R \oplus N_{3}$ \\
\hline $\mathrm{I}-2$ & $A(a, b, v),\left\{\begin{array}{l}a(v)=v_{3}, \\
b(v)=-v_{3}\end{array}\right.$ & $R \oplus N_{3}$ \\
\hline $\mathrm{I}-3$ & $A(a, b, v),\left\{\begin{array}{l}a(v)=v_{3} \\
b(v)=0\end{array}\right.$ & $R \oplus N_{3}$ \\
\hline II-1 & $A(a, b, v),\left\{\begin{array}{l}a(v)=v_{2}+v_{4}+t v_{3}, \\
b(v)=-v_{2}-v_{4}, t \geq 0\end{array}\right.$ & $N_{4}$ \\
\hline II-2 & $A(a, b, v),\left\{\begin{array}{l}a(v)=-v_{2}+v_{4}+t v_{3} \\
b(v)=-v_{2}+v_{4}, t \geq 0\end{array}\right.$ & $N_{4}$ \\
\hline II-3 & $A(a, b, v),\left\{\begin{array}{l}a(v)=v_{2} \\
b(v)=v_{3}\end{array}\right.$ & $N_{4}$ \\
\hline III-1 & $B(a, v), a(v)=t v_{2}+v_{4}, t \in R$ & $R \oplus\left\{R^{2} \rtimes R\left(\begin{array}{cc}1 & 0 \\
0 & -1\end{array}\right)\right\}$ \\
\hline III-2 & $C(a, v), a(v)=t\left(v_{1}-v_{3}\right), t>0$ & $R \oplus\left\{R^{2} \rtimes R\left(\begin{array}{cc}0 & 1 \\
-1 & 0\end{array}\right)\right\}$ \\
\hline
\end{tabular}

The type of $L(H)$ and the parameter $t$ determine the equivalence classes uniquely.

3. $\Gamma$ is virtually solvable. A group with a solvable subgroup of finite index is called virtually solvable.

(3.1) THEOREM. If $\Gamma \subset R^{4} \rtimes \mathrm{SO}(2,2)$ and $\Gamma$ acts freely and properly discontinuously on $R^{4}$ with compact quotient, then $\Gamma$ is virtually solvable.

Proof. Let $\pi=P_{l}(\Gamma)$ and $A(\pi)$ be the algebraic hull of $\Gamma$. The identity component $A_{0}$ is of finite index in $A(\pi)$. We will show $A_{0}$ is solvable. The following lemma is due to $\mathrm{D}$. Fried.

(3.2) Lemma. If $A_{0}$ fixes a vector $v \in R^{4}$ s.t. $Q(v, v) \neq 0$, then $A_{0}$ is solvable.

For a proof, cf. [3].

Assume that $A_{0}$ is not solvable. As in (1.7.2), for every $g \in A(\pi)$, $\operatorname{det}(g-I)=0$. This shows $\operatorname{det}=0$ on $L\left(A_{0}\right)$ and $\operatorname{dim} A_{0}<$ $\operatorname{dim} \mathrm{SO}(2,2)$. So $A_{0}$ contains a semisimpleconnected subgroup $S$ 
such that $\operatorname{dim} S=3$ and $L(S) \simeq \operatorname{sl}_{2}(R)$. By (1.6.2) det $\neq 0$ on $l_{i}$ so $L(S) \neq L_{i}, i=1,2$. So $L(S)$ must be a maximal subalgebra of so $(2,2)$, so $A_{0}=S$. Let $P_{i}: L(S) \rightarrow L_{i}, i=1,2$ be the projection map, then $P_{i}(L(S))=L_{i}, i=1,2$.

(3.3) Claim. There is a nonzero vector $v \in R^{4}$ such that

(i) $Q(v, v) \neq 0$;

(ii) $A_{0}(v)=v$.

To prove the claim, let $0 \neq X \in L\left(A_{0}\right)$ such that $R X$ is a split Cartan subalgebra of $L\left(A_{0}\right)$. Then $h=P_{1}(R X) \oplus P_{2}(R X)$ is a split Cartan subalgebra of $\mathrm{so}(2,2)$. By $(1.6 .3) h$ is conjugate under $\mathrm{O}(2,2)$ to $\{\operatorname{diag} \cdot(a, b,-a,-b) ; a, b \in R\}$. Since $\operatorname{det} X=0$ we can rescale and permute coordinates so $X=\operatorname{diag} \cdot(1,0,-1,0)$. Let $\{X, Y, Z\}$ be the basis of $L(A)$ such that $[X, Y]=2 Y,[X, Z]=-2 Z,[Y, Z]$ $=X$ and $X=\operatorname{diag} \cdot(1,0,-1,0)$. Then ad $X$ has three real eigenvalues on $\operatorname{so}(2,2):\{2,0,-2\}$. Let $E_{\lambda}$ be the corresponding eigenspaces, then

$$
\begin{aligned}
E_{2} & =\left\{\left(\begin{array}{cccc}
0 & c & 0 & e \\
0 & 0 & -e & 0 \\
0 & 0 & 0 & 0 \\
0 & 0 & -c & 0
\end{array}\right) ; c, e \in R\right\}, \\
E_{-2} & =\left\{\left(\begin{array}{cccc}
0 & 0 & 0 & 0 \\
d & 0 & 0 & 0 \\
0 & f & 0 & -d \\
-f & 0 & 0 & 0
\end{array}\right) ; d, f, \in R\right\}, \quad \text { and } \\
{\left[E_{2}, E_{-2}\right] } & =\left\{\left(\begin{array}{ccc}
c d-e f & -c d+e f & 0 \\
& c d+e f
\end{array}\right) ;\right. \\
0 & c, d, e, f \in R\} .
\end{aligned}
$$

So there are $c, e, d, f \in R$ such that

$$
Y=\left(\begin{array}{cccc}
0 & c & 0 & e \\
0 & 0 & -e & 0 \\
0 & 0 & 0 & 0 \\
0 & 0 & -c & 0
\end{array}\right), \quad Z=\left(\begin{array}{cccc}
0 & 0 & 0 & 0 \\
d & 0 & 0 & 0 \\
0 & f & 0 & -d \\
-f & 0 & 0 & 0
\end{array}\right)
$$


and $[X, Z]=X$ implies

$$
\left\{\begin{array}{l}
c d-e f=1 \\
c d+e f=0
\end{array}\right.
$$

i.e. $c d=-e f=\frac{1}{2}, c d e f \neq 0$. Let $v=\frac{1}{c} e_{2}-\frac{1}{e} e_{4}$. It's easy to check that $Q(v, v)=\frac{1}{c e} \neq 0, A_{0}(v)=v$.

Combining (3.3) with Lemma (3.2), we have a contradiction, so $A_{0}$ must be solvable.

4. Proof of Theorem (1.2). The principal tool is the following theorem from [4].

(4.1) THEOREM (Fried and Goldman). Let $\Gamma \subseteq \operatorname{Aff}(n)$ be virtually polycyclic and suppose that $\Gamma$ acts properly discontinuously on $R^{n}$. Then there exists at least one subgroup $H \subseteq \operatorname{Aff}(n)$ containing $\Gamma$ such that:

(a) $H$ has finitely many components and each component meets $\Gamma$;

(b) $H / \Gamma$ is compact;

(c) $H$ and $\Gamma$ have the same algebraic hull in $\operatorname{Aff}(n)$;

(d) if $\Gamma$ has a subgroup $\Gamma_{1}$ of finite index such that every element of $P_{l}\left(\Gamma_{1}\right)$ has all real eigenvalues, then $H$ is uniquely determined by the above conditions;

(e) the identity component $H_{0}$ of $H$ acts simply transitively on $R^{n}$ and $H_{0} \cap \Gamma$ is a discrete cocompact subgroup of $H_{0}$ and is of finite index in $\Gamma$.

Such a subgroup $H$ in (4.1) is called a crystallographic hull for $\Gamma$. Since a discrete solvable subgroup of Lie with finitely many components is polycyclic and we proved in $\S 3$ that $\Gamma$ in (1.2) is virtually solvable, by (4.1) we need only to check for the uniqueness of $H$. By (4.1)-(d), we need only to show that $P_{l}(\Gamma)$ has a subgroup of finite index with real eigenvalues only. Since $H_{0}$ must occur in our table of simply transitive motions and all these simply transitive mo tions, except Type III-2, have linear parts with only real eigenvalues, we need only to check Type III-2. By Bieberbach's theorem (cf. [8]), any discrete subgroup of Type III- 2 meets $T$ in a subgroup of finite index. 


\section{Classification of $\Gamma$.}

(5.1) LEMMA. Let $\Gamma$ be a uniform lattice in a simply transitive group $H \subseteq R^{4} \rtimes \mathrm{SO}(2,2)$. Then $H$ is the identity component of the crystallographic hull of $\Gamma$ if and only if $H$ is not of Type III-2.

Proof. If $H$ is of Type III-2, then $\Gamma$ has a subgroup of finite index, say $\Gamma_{1}$, such that $\Gamma_{1} \subset T$. So $\Gamma$ is virtually abelian. By [4], the crystallographic hull of a virtually abelian affine polycyclic group is itself virtually abelian, so $H$ doesn't arise from any $\Gamma$.

In the unipotent cases, the algebraic hull of $H$ is $H$ itself. So $A(\Gamma)$, the algebraic hull of $\Gamma$, is contained in $H$. Since $H_{0}^{\prime}$, the identity component of the crystallographic hull $H^{\prime}$ of $\Gamma$, acts simply transitively on $R^{4}$, the dimension of $H_{0}^{\prime}$ must be four, and then by (4.1)-(C) we have

$$
H_{0}^{\prime} \subseteq H^{\prime} \subseteq A\left(H^{\prime}\right)=A(\Gamma) \subseteq H .
$$

So $H=H_{0}^{\prime}$; then $H^{\prime}=H$.

The only remaining case is Type III-1. Since $\Gamma$ is not unipotent, $H_{0}^{\prime}$, the identity component of the crystallographic hull $H^{\prime}$ of $\Gamma$, must be nonunipotent solvable, i.e. $H_{0}^{\prime}$ is of Type III-1 and $\Gamma \subseteq H \cap H_{0}^{\prime}$. Then it's easy to show that $H_{0}^{\prime}=H$.

(5.2) COROLLARY. Up to finite covers, every flat compact complete space-form with metric of signature $(2,2)$ is of the form $H / \Gamma$, where $H$ is a simply transitive subgroup of $R^{4} \times \mathrm{SO}(2,2)$ of Type 0 , Type $\mathrm{I}$, Type II or Type III-1 and $\Gamma$ is a uniform lattice of $H$.

(5.3) Uniform lattices. The uniform lattices depend only on the structure of $H$ as a Lie group and do not depend on its embedding in $R^{4} \rtimes \mathrm{SO}(2,2)$. Since Type $0 \simeq R^{4}$, Type $\mathrm{I} \simeq R \times \mathrm{Nil}^{3}$, Type II $\simeq \mathrm{Nil}^{4}$ and Type III-1 $\simeq R \times\left\{R^{2} \rtimes\left(\begin{array}{cc}e^{t} & 0 \\ 0 & e^{-t}\end{array}\right) ; t \in R\right\}$, as Lie groups, they are exactly the same group as that listed in [8], and D. Fried gave a list of their uniform lattices there. C. T. C. Wall also studied them, cf. [7]. Here we only write them down to complete our classification.

(5.3.1) The uniform lattices of $H$ are semidirect products $Z^{3} \rtimes Z_{A}$, where $A \in \mathrm{SL}(Z)$ has a characteristic polynomial

$$
\operatorname{det}(t-A)=(t-1)\left(t^{2}-b t+1\right),
$$


where $b \geq 2$ is an integer, and $A$ and $b$ satisfy:

(i) Type 0: $A=I, b=2$;

(ii) Type I: $(A-I)^{2}=0, A \neq I, b=2$;

(iii) Type II: $(A-I)^{2} \neq 0,(A-I)^{3}=0, b=2$;

(iv) Type III-1: $b \geq 3$.

(Cf. [3] and [7] for a proof.)

Acknowledgment. The author wishes to thank Professor Ravi S. Kulkarni whose guidance and encouragement made the work of this paper possible.

\section{REFERENCES}

[1] L. Auslander, Simply transitive groups of affine motions, Amer. J. Math., 99 (1977), 809-821.

[2] A. Borel, Linear Algebraic Groups, Benjamin, New York, 1969.

[3] D. Fried, Flat spacetimes, J. Differential Geometry, 26 (1987), 385-396.

[4] D. Fried and W. Goldman, Three-dimensional affine crystallographic groups, Adv. in Math., 47 (1983), 1-49.

[5] B. Kostant and D. Sullivan, The Euler characteristic of an affine space form is zero, Bull. Amer. Math. Soc., 81 (1975), 937-938.

[6] J. Milnor, On fundamental groups of complete affinely flat manifolds, Adv. in Math., 525 (1977), 178-187.

[7] C. T. C. Wall, Geometric structures on compact complete analytic surfaces, Topology, 25 No. 2, (1986), 119-153.

[8] H. Zassenhaus, Beweis eines Satzes uber diskrete Grupen, Abh. Math. Sem. Hamb. Univ., 12 (1938), 289-312.

Received September 13, 1988 and in revised form February 27, 1989. Partially supported by the Max-Planck-Institut fur Mathematik, Bonn, West Germany and an NSF grant.

Queens College of CUNY

FLUSHING, NY 11367-0904 


\section{PACIFIC JOURNAL OF MATHEMATICS EDITORS}

\author{
V. S. VARADARAJAN \\ (Managing Editor) \\ University of California \\ Los Angeles, CA 90024-1555-05 \\ Herbert Clemens \\ University of Utah \\ Salt Lake City, UT 84112 \\ THOMAS ENRIGHT \\ University of California, San Diego \\ La Jolla, CA 92093
}

R. FINN

Stanford University

Stanford, CA 94305

HeRmanN FlaschKa

University of Arizona

Tucson, AZ 85721

VAUGHAN F. R. JONES

University of California

Berkeley, CA 94720

STEVEN KERCKHOFF

Stanford University

Stanford, CA 94305
C. C. MOORE

University of California

Berkeley, CA 94720

Martin ScharlemanN

University of California

Santa Barbara, CA 93106

\section{HAROLD STARK}

University of California, San Diego La Jolla, CA 92093

\section{ASSOCIATE EDITORS}
R. ARENS
E. F. BECKENBACH
B. H. NeumanN
F. WolF
K. YoshidA
(1906-1982)

(1904-1989)
TIONS

UNIVERSITY OF ARIZONA

UNIVERSITY OF BRITISH COLUMBIA

CALIFORNIA INSTITUTE OF TECHNOLOGY

UNIVERSITY OF CALIFORNIA

MONTANA STATE UNIVERSITY

UNIVERSITY OF NEVADA, RENO

NEW MEXICO STATE UNIVERSITY

OREGON STATE UNIVERSITY
UNIVERSITY OF OREGON

UNIVERSITY OF SOUTHERN CALIFORNIA

STANFORD UNIVERSITY

UNIVERSITY OF HAWAII

UNIVERSITY OF TOKYO

UNIVERSITY OF UTAH

WASHINGTON STATE UNIVERSITY

UNIVERSITY OF WASHINGTON 


\section{Pacific Journal of Mathematics}

\section{Vol. 148, No. $1 \quad$ March, 1991}

David Marion Arnold and Charles Irvin Vinsonhaler, Duality and

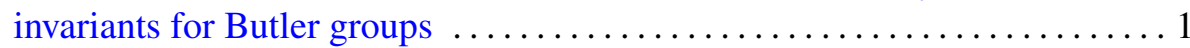

Philippe Delanoë, Obstruction to prescribed positive Ricci curvature . . . . . 11

María J. Druetta, Nonpositively curved homogeneous spaces of dimension

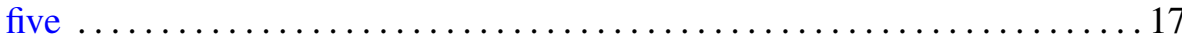

Robert Fitzgerald, Combinatorial techniques and abstract Witt rings III .... 39

Maria Girardi, Dentability, trees, and Dunford-Pettis operators on $L_{1} \ldots \ldots 59$

Krzysztof Jarosz, Ultraproducts and small bound perturbations $\ldots \ldots \ldots \ldots 81$

Russell David Lyons, The local structure of some measure-algebra homomorphisms .................................. 89

Fiona Anne Murnaghan, Asymptotic behaviour of supercuspidal characters of $p$-adic $\mathrm{GL}_{3}$ and $\mathrm{GL}_{4}$ : the generic unramified case $\ldots \ldots \ldots \ldots \ldots 107$

H. Rouhani, Quasi-rotation $C^{*}$-algebras $\ldots \ldots \ldots \ldots \ldots \ldots \ldots \ldots \ldots \ldots \ldots \ldots \ldots \ldots$

Ignacio Sols Lucía, Michał Szurek and Jaroslaw Wisniewski, Rank-2

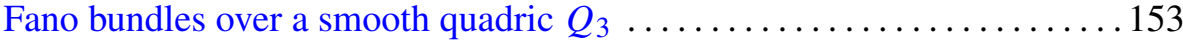

Martin Strake and Gerard Walschap, Ricci curvature and volume

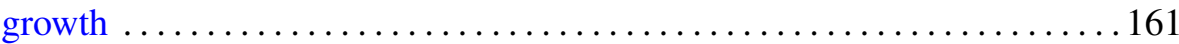

Anton Ströh and Johan Swart, A Riesz theory in von Neumann algebras . . 169

Ming Wang, The classification of flat compact complete space-forms with metric of signature $(2,2)$ 